\title{
POST-SCARLATINAL DIPHTHERIA.
}

\author{
(One Figure.)
}

\author{
By W. T. GORDON PUGH, M.D., B.S. (LoND.), \\ Senior Assistant Medical Officer, North-Eastern Hospital, Metropolitan \\ Asylums Board, London.
}

\section{The Statistics of Post-scarlatinal Diphtheria.}

For several years returns have been made by the hospitals of the Metropolitan Asylums Board on the subject of this complication, and these are here briefly analysed.

The period under observation may conveniently be divided into two parts, the first including the years prior to the introduction of the bacteriological method of diagnosis and to the use of antitoxin in these hospitals, the second the years subsequent. Previous to 1895 only cases of scarlet fever which showed clinical diphtheria, having membrane in the fauces or exhibiting laryngeal symptoms, were designated postscarlatinal diphtheria; since that year all cases of secondary throat illness associated with the diphtheria bacillus have been returned as diphtherial, including those which would from the clinical appearance alone have been regarded as simple tonsillitis. It is probable that the majority of the cases which are now seen in these hospitals would formerly have been described as tonsillitis. The significance of this is shown in the fact that only 11.9 per cent. of the cases recorded in the years 1896 1900 presented laryngeal symptoms, whereas in six years belonging to the former period, 1889-1894, laryngeal diphtheria formed no fewer than $52 \cdot 8$ per cent. of the cases occurring at those hospitals which made a return of the character of the cases. It will thus be seen that the first half of each of the two tables that follow is to be regarded as on a totally different footing from the second.

It was, therefore, natural that in the year 1895 there should appear a sudden and large increase in the incidence of secondary diphtheria 
among the patients in the scarlet fever wards of these hospitals. It would seem probable, however, in view of the early recognition of

Table I.

\begin{tabular}{l|c|c|c|c|c|c|c|c|c|c}
\hline & 1891 & 1892 & 1893 & 1894 & 1895 & 1896 & 1897 & 1898 & 1899 & 1900 \\
\hline $\begin{array}{c}\text { Searlet Fever cases } \\
\text { completed }\end{array}$ & 5,444 & 11,326 & 14,867 & 12,637 & 10,422 & 15,054 & 15,250 & 12,771 & 13,327 & 10,749 \\
$\begin{array}{c}\text { Cases of post-scar- } \\
\text { latinal diphtheria }\end{array}$ & 99 & $\mathbf{2 1 7}$ & $\mathbf{2 0 7}$ & $\mathbf{2 1 0}$ & 453 & 705 & 796 & 661 & 692 & 405 \\
$\begin{array}{c}\text { Percentage inci- } \\
\text { dence }\end{array}$ & $1 \cdot 8$ & $1 \cdot 9$ & $1 \cdot 3$ & $1 \cdot 6$ & $4 \cdot 3$ & $4 \cdot 6$ & $5 \cdot 2$ & $5 \cdot 1$ & $5 \cdot 1$ & $3 \cdot 7$ \\
\hline
\end{tabular}

atypical cases of diphtheria by bacteriological means and their consequent prompt isolation, that, in spite of the figures, the number of patients actually infected with this disease has in reality very considerably diminished in recent years.

The influence of the change in the basis of diagnosis and the introduction of a new method of treatment is especially noticeable in Table II., which shows the deaths among patients who have suffered from post-scarlatinal diphtheria. It must, however, be pointed out that

Table II.

\begin{tabular}{|c|c|c|c|c|c|c|c|c|c|c|}
\hline & 1891 & 1892 & 1893 & 1894 & 1895 & 1896 & 1897 & 1898 & 1899 & 1900 \\
\hline $\left.\begin{array}{c}\text { Cases of post-scarlatinal } \\
\text { diphtheria }\end{array}\right\}$ & 89 & 217 & 207 & 210 & 453 & 705 & 796 & 661 & 692 & 405 \\
\hline Deaths among these & 55 & 95 & 120 & 77 & 67 & 36 & ao & 24 & 25 & 12 \\
\hline
\end{tabular}

these are deaths among patients who have died at any period during their stay in hospital subsequent to an attack of secondary diphtheria, and are not necessarily deaths due to this complication. Thus, in the year 1901, when special attention was paid to this point, of 23 deaths following an attack of post-scarlatinal diphtheria, 12 are stated to be from causes unconnected with the diphtheria. The excess of deaths recorded in the table over the deaths really to be attributed to the complication was probably not a very material one while the diagnosis was made on clinical grounds; since 1895, it is obvious, the influence of this system of registration has become much more marked.

For these several reasons it is impossible to arrive at the mortality with correctness. The case death-rate, and even that calculated on the 
number of scarlet fever patients treated, are both largely in excess of the true figures.

With the terrible mortality among patients attacked by this complication in the years before the introduction of antitoxin-when diphtheria assisted in producing the fatal issue in over 12 per cent. of the scarlet fever deaths-there may be happily contrasted the results obtained at this hospital in 1901, when of 3,094 scarlet fever patients under treatment only one died who had suffered from secondary diphtheria, even this death being attributable to lobar pneumonia occurring a month after the attack. There are, perhaps, few examples of advance in medical science so striking as the extraordinary reduction in mortality from post-scarlatinal diphtheria.

Sex distribution.-The number of female patients under treatment for scarlet fever, during the five years of which complete statistics are available, was not greatly in excess of the males,- $-32,395$ males; 34,458 females. It will be seen, however, in Table III. that the number of

Table III. Sex distribution in the five years, 1896-1900.

\begin{tabular}{|c|c|c|c|c|c|c|}
\hline & \multicolumn{2}{|c|}{ All cases } & \multicolumn{2}{|c|}{ Faucial and Nasal } & \multicolumn{2}{|c|}{ Laryngeal } \\
\hline & Males & Females & Males & Females & Males & Females \\
\hline $\begin{array}{c}\text { Cases of post-scarlatinal diph- } \\
\text { theria }\end{array}$ & 1,520 & 1,739 & 1,297 & 1,571 & 223 & 168 \\
\hline Deaths among these $\ldots$ & 65 & 62 & 36 & 44 & 29 & 18 \\
\hline
\end{tabular}

females developing secondary diphtheria was, proportionately, considerably larger. This increase agrees with the sex distribution among patients suffering from ordinary diphtheria, the number admitted during the same five years being 15,437 males; 17,856 females. The large percentage of males among those with laryngeal symptoms is striking.

Age distribution.-In connection with this point it should be mentioned that children under three years of age are not usually transferred to the convalescent hospitals, in which the incidence of this complication is considerably higher than at the acute hospitals.

The greatest attack rate is in the fifth year; in the diphtheria notifications in London, per 1,000 living, also, this age is pre-eminent. An age-period curve would follow the same general course as the curve for diphtheria notifications, but the rise and fall would be much more gradual and the curve flattened out. 
TABLE IV. Age distribution in the five years, 1896-1900.

\begin{tabular}{c|c|c|c|c|c||c|c|c|c}
\hline & $\begin{array}{c}\text { under } \\
1\end{array}$ & $1-2$ & $2-3$ & $3-4$ & $4-5$ & $5-10$ & $10-15$ & $15-20$ & $\begin{array}{c}\text { over } \\
20\end{array}$ \\
\hline $\begin{array}{c}\text { Cases of post-scarla- } \\
\text { tinal diphtheria }\end{array}$ & $\mathbf{2 0}$ & $\mathbf{7 5}$ & 182 & $\mathbf{3 9 2}$ & $\mathbf{4 7 1}$ & $\mathbf{1 , 5 4 1}$ & $\mathbf{4 5 2}$ & $\mathbf{8 9}$ & $\mathbf{3 7}$ \\
$\begin{array}{c}\text { Deaths among these ... } \\
\begin{array}{c}\text { Total scarlet fever } \\
\text { admissions }\end{array}\end{array}$ & $\mathbf{7}$ & 19 & $\mathbf{1 7}$ & $\mathbf{2 7}$ & $\mathbf{2 2}$ & $\mathbf{3 1}$ & $\mathbf{4}$ & - & - \\
$\begin{array}{c}\text { Percent. incidence of } \\
\text { post-scarl. diphtheria }\end{array}$ & $3 \cdot 1$ & 2,274 & 4,417 & 6,314 & 6,958 & 26,740 & 12,080 & 3,891 & 3,545 \\
\hline
\end{tabular}

Seasonal infuence.-If the number of cases of post-scarlatinal diphtheria, developing in each month in the five years under consideration, be averaged, and corrected for the mean daily number of scarlet fever patients under treatment in each of these months, it will be

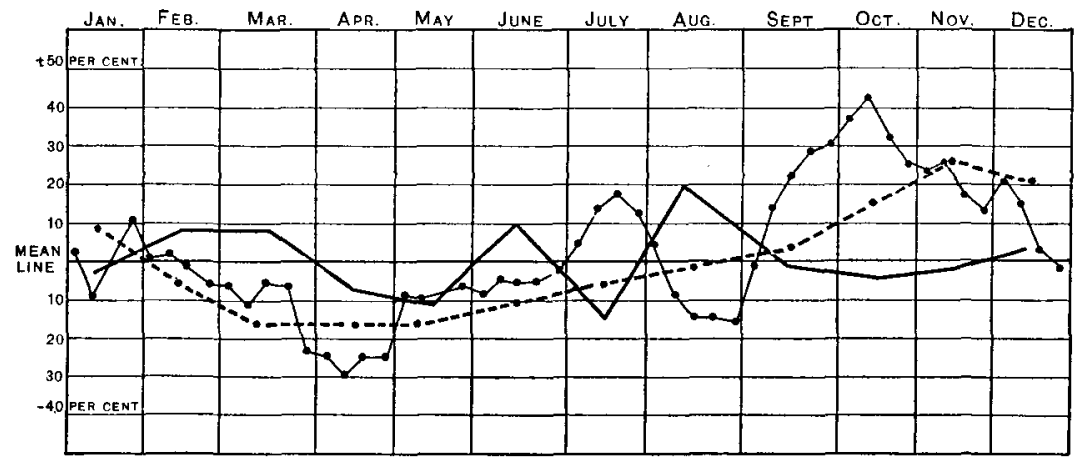

Dotted line indicates average daily number of scarlet fever patients under treatment.

Thick line indicates mean percentage incidence of post-scarlatinal diphtheria, calculated on the average daily number of scarlet fever patients under treatment.

Thin line indicates the weekly number of diphtheria notifications in London.

The mean line represents $2,721,1.99$ and 1,178 , respectively. The chart deals with the five years, $1896-1900$.

found that the incidence of this complication does not follow the seasonal variation of diphtheria in the Metropolis, nor does it appear to depend on whether the hospitals are full or the reverse. These points are set forth in the accompanying chart. It must be noticed, however, that the statistics deal only with 5 years and 3,259 cases of postscarlatinal diphtheria, numbers, perhaps, not sufficiently large to allow of accurate deduction. 
Incidence at acute and convalescent hospitals.-In calculating the incidence of post-scarlatinal diphtheria on the number of patients discharged and dead an important correction is necessary. Returns made in the years 1899 and 1900 show that the patients treated to recovery or death in the Board's town institutions have been in hospital about 68 days on an average, while patients who have completed their recovery or died at the convalescent hospitals have been, on an average, 31 days at the town hospital and about 4.8 days at the convalescent institution. If the calculation be made on "patient-days" (on the

Table V.

Incidence at Acute and Convalescent Hospitals, 1896-1900.

\begin{tabular}{|c|c|c|}
\hline & $\begin{array}{c}\text { Acute } \\
\text { Hospitals }\end{array}$ & $\begin{array}{c}\text { Convalescent } \\
\text { Hospitals }\end{array}$ \\
\hline Searlet fever cases discharged to their homes or dead & 33,296 & 33,855 \\
\hline Cases of post-scarlatinal diphtheria among these $\ldots \ldots$ & 1,510 & 1,749 \\
\hline
\end{tabular}

"foot-pound" principle), it will be found that the liability to postscarlatinal diphtheria at the convalescent hospitals is about two and a third times as great as at the town hospitals.

\section{The Origin of Post-scarlatinal Diphtheria.}

I now propose to consider the principal reasons which have been suggested for the occurrence of secondary diphtheria in scarlet fever wards.

(i) Sanitary defects.-As advances have been made in our knowledge of the bacterial origin of diphtheria, the belief, once generally held, that defective drainage played an important part in disseminating this disease has gradually waned. However, it may be well to recall that Sweeting ${ }^{1}$, in 1893, investigated this point in connection with the Board's hospitals, and found that post-scarlatinal diphtheria had prevailed in like degree in hospitals with ventilated and in those with unventilated soil-pipes; in hospitals with automatic flushing apparatus, and in hospitals without such appliances; in hospitals with elaborate systems of ventilation and disconnection, and in hospitals where these were of the most meagre and incomplete kind. In fact, the diversity

1 Trans. of Epidem. Soc., Lond., xII. 
was so great that no common factor of drainage defect could be pointed to as explaining the long-continued yearly recurrence of this condition of post-scarlatinal diphtheria.

(ii) The treatment in the same hospital of the two diseases.-It is but natural that a layman, unacquainted with the administration of a fever hospital, should, when he hears that his child, convalescent from scarlet fever, has developed diphtheria, forthwith conclude that infection has been derived from cases of diphtheria treated in the same hospital. This opinion has to some extent been shared by members of our own profession. Thus, Sweeting apparently believed that there was a connection between the reception of both diseases in the Board's hospitals and the incidence of post-scarlatinal diphtheria. $\mathrm{He}$ concluded from a study of his statistics that "there had been a marked increase of the complication at the acute hospitals since diphtheria was received, although it had undoubtedly existed to a minor extent at some of them before diphtheria was admitted," but "...that at the Northern Convalescent Hospital it had existed before and after the reception of diphtheria convalescents, and that its prevalence had apparently been inappreciably affected thereby."

Now if, as the supporters of this theory have held, the treating in the same hospital of the two diseases is the main cause of postscarlatinal diphtheria, one would expect it to be of comparatively rare occurrence in hospitals reserved entirely for the treatment of scarlet fever. That this is not so is evident from the fact that 160 cases were recorded for this (the North-Eastern) hospital during the five years, 1896-1900, during which period only patients certified to be suffering from scarlet fever were received. Similarly at Gore Farm, which up to 1899 received scarlet fever convalescents only, 273 cases of secondary diphtheria occurred during the two years 1897 and 1898 .

It would be interesting to compare the incidence of post-scarlatinal diphtheria at hospitals receiving scarlet fever only and at those admitting both diseases. In the case of the acute hospitals such comparison would, however, be without value, on account of the varying proportion of patients transferred to the convalescent institutions. Comparison of the latter hospitals is free from this particular objection:-at Gore Farm during the years 1896-98, a period when it received scarlet fever convalescents only, 4.5 per cent. of the patients developed secondary diphtheria; at the Northern Hospital, admitting convalescents from both diseases, the almost identical percentage incidence of 4.9 is recorded during the same three years. 
It may, therefore, I think, be regarded as proved, so far as statistics are able to help one, that the aggregation upon the same site of the two diseases is not an important factor in the etiology of post-scarlatinal diphtheria. Is it possible for such association ever to give rise to this complication? Goodall ${ }^{1}$ in $\mathbf{1 8 9 6}$, after pointing out certain fallacies in Sweeting's statistics, said he had not been able to satisfy himself that, save in very exceptional instances, infection had been conveyed from the diphtheria to the scarlet fever wards. Indeed, since diphtheria spreads solely through intimate contact with the source of infection, it can extend to the scarlet fever wards only in consequence of imperfect separation of the convalescents or through conveyance there by members of the staff. I am not aware that the first means of infection exists at any hospital and will, therefore, confine my remarks to the second.

Practically the only persons involved are the medical officers and the nurses. The former, however, are not brought into sufficiently close contact with their patients to encourage the belief that they serve in any degree of frequency as sources of infection. The intimate relations, on the other hand, existing between a nurse and the children under her care render her more likely to prove an important factor in the spread of this disease. On a later page the liability of a nurse in close attendance on diphtheria patients to acquire virulent bacilli will be pointed out. Is there any evidence that a nurse, in the best of health herself, can by this means convey infection? Proof has before now been furnished, but the following instance seems of sufficient interest to deserve mention.

Prior to the opening of our diphtheria wards there was an isolation building in this hospital, used for cases erroneously diagnosed as scarlet fever, containing four separate rooms, which were looked after by a single nurse. In one was a child with bronchitis; in another a patient suffering from diphtheria. The latter died on November 18th, five days after admission. On December 3rd the bronchitic child, who had not yet left his bed, developed laryngeal diphtheria necessitating tracheotomy. No source of infection appeared possible save by the medical or nursing staff. Cultures were made from the throats of all who had been in contact with the child, and from one nurse, who had been in attendance on the diphtheria case of a fortnight before, virulent Klebs-Loeffler bacilli were obtained. She had throughout had no sore throat, and the tonsils showed only chronic enlargement.

1 Trans. of Epidem. Soc., Lond., xv. 
This subject has been dealt with at some length because it appears to be the only conceivable way by which the disease can be conveyed from the diphtheria to the scarlet fever wards.

(iii) The introduction of unrecognised diphtheria.-That this is the usual source of infection there can be little doubt. It is interesting to note how the yearly incidence of post-scarlatinal diphtheria in fever hospitals has varied with the prevalence of diphtheria outside. Thus Meredith Richards ${ }^{1}$ stated that in 1893, 1894 and the first half of 1895 there were no cases of this complication noted at the Birmingham Fever Hospital, into which diphtheria was not admitted. In July, 1895, fatal laryngeal diphtheria occurred in a convalescent child, and this was followed by secondary cases and numerous other outbreaks during the rest of the year. The diphtheria deaths recorded for Birmingham had been 43 in 1893, and 50 in 1894; in 1895 there was a sudden rise to 163 . This agrees with the experience in the hospitals of the Metropolitan Asylums Board, the incidence of post-scarlatinal diphtheria had been $0 \cdot 2,0 \cdot 4$ and $0 \cdot 4$ per cent. in $188.5-87$ respectively. In 1888 it rose to $1 \cdot 1$ per cent. This coincided with a sudden bound in the diphtheria death-rate per million in London, from 235 in 1887, to 319 in 1888. It happened that it was in the latter year that the managers first received diphtheria cases into their hospitals, but, as Goodall $^{2}$ pointed out, the increase in post-scarlatinal diphtheria was noticeable before October 23rd when the diphtheria wards were opened, and only 99 cases of the latter disease were admitted between that date and the end of the year. Thus, the suggestion that the rise was due to the reception of diphtheria patients falls to the ground.

Among the cases received into fever hospitals certified scarlet fever, a few can be readily recognised clinically as uncomplicated diphtheria. A larger class is that in which there is on admission evidence only of tonsillitis, for patients have not infrequently lost by the time they arrive at the hospital the other signs upon which the practitioner founded his diagnosis, and yet many of these are proved subsequently, by the occurrence of desquamation, to be suffering from the disease certified. Owing to the limited number of isolation rooms, a considerable proportion of these cases of apparent tonsillitis are admitted for observation into the scarlet fever wards, and one of mild diphtheria might thus be the origin of an outbreak of post-scarlatinal diphtheria. 
Another class of case is that of double infection. Occasionally, in addition to the signs of scarlet fever, the patient presents undoubted diphtheritic membrane in the throat. These cases, however, must not be confounded with a much larger number in whom a condition of throat more or less simulating diphtheria is found; cultures, as a rule, show absence of the specific bacillus, but sometimes organisms are found which morphologically are indistinguishable from it. It will be shown later that non-virulent diphtheria bacilli are not uncommon in normal and scarlatinal throats; hence, in the absence of definite membrane, the mere finding of the bacillus, without recourse to inoculation, cannot be regarded, in this throat condition, as proof of the co-existence of the two diseases. However, it cannot be denied that cases of the combined diseases may occasionally be admitted in which the local evidence of diphtheria is so slight, or else so masked by the lesions of scarlet fever, as to escape recognition. Finally, as is well known, there may, under certain circumstances, be present in throats which are apparently quite healthy, virulent bacilli which are capable of causing diphtheria in other patients.

So far I have dealt only with the conveyance of infection in the throat. There is another source of infection which is, I believe, of almost equal importance, the nose. A disease which is known to rhinologists as fibrinous rhinitis, but which I see no logical objection to calling nasal diphtheria, appears to be not at all uncommon among children. Producing, as it does, little or no constitutional disturbance and no external evidence save discharge and soreness-and even these may be almost absent-it is a disease which is easily overlooked.

The whole subject of the presence of diphtheria bacilli in the throat and nasal cavities, and of the significance of these organisms under various conditions, is of such vast importance in dealing not only with post-scarlatinal diphtheria, but also with outbreaks in institutions and towns, that it has been thought well to enter into it in considerable detail in the following sections, in order that the limitations which exist to the usefulness of bacteriology in such endeavours may be the better appreciated.

\section{Diphtheria Bacilli in the Throat.}

An examination of the literature of diphtheria will reveal the fact that considerable lack of uniformity has existed in describing and naming diphtheroid organisms, a fact which depreciates the value of many of the observations. In the following consideration of the 
occurrence of diphtheria bacilli under various conditions, $I$ have selected for illustration investigations in which there has apparently been adopted the classification which is now in general use, founded on one suggested by Park and Beebe ${ }^{1}$ in 1894, namely, (i) the virulent diphtheria bacillus (Klebs-Loeffler), (ii) the non-virulent diphtheria bacillus, and (iii) the Hoffmann bacillus. It is unnecessary for the purpose of this paper that the methods of distinguishing between the diphtheria bacillus and that of Hoffmann should be entered into in detail, but it may be stated brietly that, to confirm the microscopical differentiation, Neisser's double stain and the reaction produced by growth in neutral litmus glucose broth are, apart from tests of virulence, the chief methods relied on.

By the vast majority of bacteriologists it is believed that only bacilli of the first group are capable of causing diphtheria. By some writers, however, Hoffmann's bacillus is said to cause a mild tonsillitis, but I have never been able to convince myself that, in any of the numerous cases of tonsillitis which have occurred at this hospital, its presence was ever more than accidental. As will be shown later a considerable percentage of children have normally these bacilli in nose or throat. By a few observers the possibility of the conversion of Hoffmann's bacillus into the virulent diphtheria bacillus has been asserted, but the evidence of this cannot be regarded as satisfactory.

(i) In scarlet fever patients on admission into hospital.-A series of 420 unselected cases received into this hospital, certified scarlet fever, were examined as to the presence of bacilli in the throat on admission, the cultures being taken in the receiving room to avoid complication. The inoculations were kindly performed by Dr Cartwright Wood.

The cases may be described in relation to clinical diphtheria.

Two were uncomplicated faucial diphtheria. One was easily recognizable as such; the other presented follicular deposit only. A culture from the latter was tested by inoculation and the bacilli were found to be virulent.

One case was scarlet fever associated with a croupy cough and considerable obstruction to respiration. Diphtheria bacilli were obtained from the fauces, which were inflamed but without deposit.

Two were scarlet fever complicated by fibrinous rhinitis, the presence of which was recognized in the receiving room. From the throats of both these patients diphtheria bacilli were obtained, although there was clinically only the inflammation of scarlet fever.

\footnotetext{
1 New York Medical Record, 29th Sept. 1894.
} 
In 17 of the remaining 415 cases was the diphtheria bacillus found. In three of these the fauces were merely congested; one culture was tested by inoculation and proved non-virulent. Nine showed inflammation without deposit; three of these were examined as to pathogenicity with negative result. Three cases presented follicular exudation. One case showed ulceration of tonsils and uvula, and one case a pultaceous mass of exudate on the tonsils with some ulceration; bacilli from the latter case were found on inoculation to be nonpathogenic to guinea-pigs. Thus, in five of the cases which clinically did not suggest being complicated with diphtheria, the inoculation test was applied with a negative result; the bacilli in each of these cultures stained with Gram's and Neisser's solutions, and rendered neutral litmus glucose broth acid.

Hoffmann's bacillus was found in 67 of the cultures.

Garratt and Washbourn ${ }^{1}$ examined the throats of 666 cases of scarlet fever admitted under their care at the London Fever Hospital from March, 1896, to December, 1898. In eight, or $1 \cdot 2$ per cent., were found bacilli morphologically resembling $B$. diphtheriae. The inoculation test was not applied; in only one case was there reason to suspect the presence of diphtheria from the clinical appearance of the throat; in another case there was a history of intimate exposure to diphtheria. It will be noticed that this percentage is considerably lower than that found in the patients admitted into this hospital. The difference is possibly dependent on the higher average age and social status of patients at the London Fever Hospital. The relative frequency among the class of patients admitted to the Board's hospitals is confirmed by an investigation of Goodall's ${ }^{2}$ in 1896, when among 87 cases of scarlet fever examined on admission six patients were found to have diphtheria bacilli of the long variety in their throats.

(ii) Among the general public.--It will be interesting now to consider the presence of these organisms among the general public. Apart from investigations in connection with outbreaks and epidemics, however, there are but few reliable accounts of the examination of healthy throats.

Theoretically, one would expect that the prevalence of the diphtheria bacillus would depend on whether diphtheria was endemic or not in the locality, that the proportion of persons involved would vary with the season, and that important factors would be the age of the persons 
examined, their social status and consequent surroundings. The results however, in limited researches, such as the following, must be subject to many variations, and no one percentage can be accepted as expressing the actual condition.

Denny', of Brookline, Mass., examined 235 healthy individuals, 216 children and 19 adults, a large proportion being of the well-to-do class. In cultures from their throats only once was the diphtheria bacillus found. This was a school-girl, who, as far as was known, had not been in contact with any case of diphtheria. The bacilli were so few that a pure culture for inoculation could not be obtained.

Park and Beebe ${ }^{2}$, of New York, on the other hand, examined 275 persons, chiefly hospital and dispensary patients, who were not known to have been exposed to infection; from the throats of 26 diphtheria bacilli were obtained, which in no fewer than 23 cases proved non-virulent to guinea-pigs. Of these persons 50 were adults, among whom non-virulent bacilli were found twice. One of the three cases with virulent bacilli was found to be from a house where a case of supposed croup had existed three weeks before.

Kober ${ }^{3}$ examined 600 school-children, whom, he says, he selected from the lowest standards because diphtheria was more common at that age. In fifteen diphtheria bacilli were found, in each case staining by Neisser's method and rendering glucose broth acid; ten, however, proved non-virulent to guinea-pigs. Of the five children with virulent bacilli, one sat at school next a child who had had diphtheria eight weeks before, three were playmates of neigbbours' children who had had diphtheria recently, and the fifth had associated with a family in which a fatal case of the same disease had occurred ten weeks before. With regard to the ten children with non-virulent bacilli, in five cases no connection with diphtheria could be traced, in four there had been diphtheria in the same or the next house, while the remaining boy had played with a child who had had this disease some time before.

Hewlett and Murray ${ }^{4}$ examined bacteriologically the throats of all the children (385) received into the Victoria Hospital for Children, London, for diseases other than diphtheria during 1900. In no fewer than 58 ( 15 per cent.) the Klebs-Loeffler bacillus is stated to have been found, in 92 (24 per cent.) the bacillus of Hoffmann. Seven of the 58

1 Boston Med. and Surg. Journ., 22nd Nov., 1900, p. 516.

${ }^{2}$ Amer. Journ. of Med. Sciences, Oct. 1894.

3 Zeitschr. f. Hygiene, 1899, Bd. xxxI., s. 433.

${ }^{4}$ Brit. Med. Journ., 15ith June, 1901, p. 1474. 
presented some clinical evidence of diphtheria; in three of these, however, it is remarked the bacillus was not found at the first examination of their throats, but they were sent to a fever hospital 5, 18, and 23 days' respectively, after admission, diphtheria bacilli being then present. Three of the cultures were tested as to virulence, but it is not related in the paper from which class of case they were obtained. Two were not lethal to guinea-pigs; the third proved virulent.

Similarly, Steenmeyer ${ }^{1}$ says that, in Rotterdam, he found in 7 per cent. of persons examined diphtheria bacilli in the normal mucous membrane of the pharynx.

(iii) Among nurses in attendance on diphtheria patients.-The wards for the treatment of diphtheria in this hospital are new, wellventilated and well-lighted, and the cubic space per patient is unusually large. The nurses have been instructed as to the dangers attached to the acquiring of diphtheria bacilli in the throat, and it is to be presumed are careful to avoid unnecessary risks. Nevertheless, in a series of single cultures made from the throats of 56 nurses working in these wards diphtheria bacilli were found in seven. One of these cultures was submitted to the inoculation test and proved virulent to the guineapig. The throats of all were clinically normal. In scarlet fever wards nurses appear to be less particular and more given to the fondling of children; and in cultures made in connection with cases of post-scarlatinal diphtheria a larger proportion of nurses have sometimes been found to harbour the bacillus. It is interesting to observe, however, that bacilli found even under these circumstances are not necessarily virulent. For instance, it happened that several cases of tonsillitis occurred in one of our convalescent scarlet fever wards. Cultures showed a rather short diphtheria bacillus, and similar bacilli were obtained from a few of the other patients with normal throats. Cultures were made from the nurses. One was found to have the same short bacillus in her throat; another had very long diphtheria bacilli of quite another type. The former developed a small patch of membrane a day or two later. It being suspected that the long bacilli of the second nurse were not connected with the outbreak, their virulence was tested;--although the bacilli showed well-marked pole granules with Neisser's reagents, and produced acid fermentation of glucose broth, a negative result followed the inoculation of a guinea-pig.

(iv) Among others who have been exposed to infection.-When cultures are made from convalescent patients occupying a scarlet

1 “Dissertation," Utrecht. Ref. Baumgarten's Jahresbericht, 1898, s. 216. 
fever ward in which a case of secondary diphtheria has occurred, it is usual to find $B$. diphtheriae in some of the normal throats. In several cases at this hospital these cultures have been tested by inoculation, and found to be virulent.

Many other investigations might be quoted proving the liability of those who are brought into close contact with patients suffering from diphtheria to acquire virulent bacilli in their throats without showing any signs of the disease.

Johannessen ${ }^{1}$ found the virulent bacillus present in the healthy throats of three out of 20 children in a ward in which a case of diphtheria had occurred.

Park and Beebe ${ }^{2}$ examined the throats of the healthy children of 14 families in which one or more of the other members had diphtheria. There were in all 48 healthy children; in 13 of the families and in 50 per cent. of the children diphtheria bacilli were found. Six cultures were tested for virulence with positive results.

$\mathrm{Kober}^{3}$ examined cultures taken, by the doctors in attendance, from the throats of 128 persons who were in contact with people ill with diphtheria. In 15 he found diphtheria bacilli, which in each case proved virulent. In 10 of the cases the throat was normal; in 5 there was slight angina.

(v) In institutions.-Goadby ${ }^{4}$ in 1898 examined bacteriologically the throats of 100 healthy children in an industrial school where no diphtheria had occurred for two years. Carbol-methylene blue and Neisser's stain -were used, and Hoffmann's bacillus differentiated. Diphtheria bacilli were found in 18 of the cultures. Whether these were of the second group, the saprophytic or non-virulent variety, was not ascertained, the inoculation test, as $\mathrm{Mr}$ Goadby has kindly informed me, not being applied. I might mention, however, that in the throat of a child admitted to the North-Eastern Hospital for scarlet fever from a large orphanage, in which no case of diphtheria had occurred for two years, acid-forming bacilli, indistinguishable from the B. diphtheriae, were obtained, which on inoculation were found to be nonpathogenic to the guinea-pig.

Goadby was at the time investigating an epidemic of diphtheria at the Poplar Union Schools, where about 600 children are kept on barrack principles, there being but one play-room for each sex.

1 Deutsche med. Woch., 1895, xxI.

2 New York Medical Record, 29th Sept., 1894.

3 loc. cit.

4 Trans. of Epidem. Soc., Lond., XIX.

Journ. of Hyg. II 
Twenty-three cases of diphtheria had already occurred when the cultures were taken. No fewer than 190 (32 per cent.) out of the 586 children examined were found to have diphtheria bacilli in their throats. What proportion of these were of the virulent variety it is impossible to say; cultures from two children, who had no clinical signs of throat affection, were found to be fully virulent. Only 15 of the 190 subsequently developed clinical diphtheria.

Park and Beebe ${ }^{1}$ examined 55 children in a foundling hospital, where from time to time cases of true diphtheria had occurred. Among them six were found to have diphtheria bacilli, five of the cultures being of the virulent variety.

Aaser $^{2}$, in an outbreak of diphtheria in a soldiers' barracks, found the bacillus in 17 out of 89 healthy throats. Denny ${ }^{3}$ in 1899 examined the throats of 200 boys in a truant-school, in which four cases of diphtheria with membrane had occurred. In 22 the cultures gave a positive result; only six of these boys had sore throats, the others being apparently quite healthy. Berry and Washbourn ${ }^{4}$ met with like results in an examination, under similar circumstances, of the throats of children at the London Orphan Asylum in 1898. In none of these investigations, however, is it stated that the virulence of the bacilli was examined.

(vi) In epidemics of diphtheria.-Cobbett ${ }^{5}$, in connection with an outbreak of diphtheria occurring among children attending several of the day-schools at Cambridge in the autumn of 1900, examined 650 persons not suffering from diphtheria, who had been directly or remotely exposed to infection, about 300 being children attending the schools or brothers and sisters of these pupils. Nineteen were found to harbour diphtheria bacilli; a few of these had slight sorethroat at the time of examination. In 8 the inoculation test was applied; 5 were found to be virulent, and 3 non-virulent.

In the Spring of 1901 there was a recrudescence of the disease at Cambridge and the investigation was resumed. Eighty-four children, attending three schools where there had been 2,1 and 0 cases, respectively, of diphtheria, were cultured with negative result. Sixtythree boys at another school with 160 scholars, where several cases of diphtheria had occurred, were examined, with the result that diphtheria bacilli were found in $10 ; 7$ of the cultures were tested by inoculation,3 proved virulent, 4 non-virulent.

I loc. cit. $\quad 2$ Deutsche med. Woch., 1895.

3 loc. cit.

+ Trans. of Epidem. Soc., Lond., xıx.

5 Journal of Hygiene, I., pp. 228, 235, and 485. 
Graham-Smith ${ }^{1}$, dealing with a similar outbreak at Colchester, cultured the healthy throats of children living in homes in which a case of diphtheria had occurred within from 3 to 4 months of the examination; 407 scholars from 19 schools, 59 persons above or below school age, and 55 persons from the Colchester Union, -519 in allwere examined. In 54, or 1044 per cent., were Klebs-Loeffler bacilli found in the throat.

In the preceding paragraphs an attempt has been made to present the circumstances and results of various investigations succinctly, but without omitting points of importance in connection with the subject under consideration. It will be interesting now to consider the significance of these bacilli. As has already been said, it is almost universally held that only those diphtheria bacilli which are pathogenic to the guinea-pig are capable of causing the disease in man. Can the virulent bacillus lose its virulence in nature? Does the non-virulent bacillus under any circumstances become virulent?

Artificially, Roux and Yersin found it was possible to produce an attenuation of the virulence of the bacillus in a number of ways?. For instance, if a current of sterile air was kept passing through a broth culture maintained at a temperature of $39 \cdot 5^{\circ} \mathrm{C}$., after about two weeks some of the bacilli began to lose their virulence, and at the end of about four weeks all the bacilli had lost their virulence and produced nonvirulent cultures; a little while after losing their virulence the bacilli remaining in the culture died. It has not yet been directly proved that such a change occurs naturally. Park ${ }^{3}$, in 1900, stated that in his experience bacilli, which at the beginning were virulent, continued virulent so long as they remained in the throat. Cobbett ${ }^{4}$ says that in seven cases where the diphtheria bacilli present in the throat were tested on from 2 to 10 occasions the virulence was found constant. It is interesting to note, on the other hand, that the non-virulent bacillus was obtained in a culture taken from a patient examined for the first time during convalescence from diphtheria. The number of experiments recorded, however, is but small, and the point can hardly be regarded as settled. That such a change does occur naturally is evident from the results of inoculation in the series of observations summarized above. Roux and Yersin found that if from time to time cultures were made

1 Journal of Hygiene, Ir., p. 170.

2 Ref. Park and Beebe, loc. cit.

3 Trans. of the Assoc. of Amer. Physicians, Xv., 1900, p. 222.

loc. cit.

21-2 
from dried bits of membrane, a period finally came when the bacilli, although alive, had become non-virulent. It is possible that this is one way in which the natural transformation of the bacillus occurs; as may be seen in the above investigations when the bacillus is acquired by infection from a patient suffering from diphtheria it is usually found to be virulent, whereas when the source of infection is remote or untraceable the bacillus is generally of the non-virulent variety. It may be added that it seems extremely probable that the non-virulent bacillus can be, as such, transmitted from one child to another.

The second question must, in the present state of our knowledge, be answered in the negative. Roux and Yersin were unable to give back virulence to those bacilli which had been completely robbed of it by the above method, or to those which had no virulence originally when obtained from the throat. Their attempts were more successful when they used a bacillus that still retained some slight action on the guinea-pig-by injecting a mixture of this non-fatal bacillus and very active cultures of the streptococcus of erysipelas, virulence was, though not invariably, restored. Shattock ${ }^{1}$ cultivated these bacilli in broth over which faecal air was passed, but no toxic properties were acquired even though the treatment was prolonged for a period of two months. Lubowski ${ }^{2}$ found he could not render the non-virulent bacillus pathogenic to guinea-pigs by repeatedly passing it through these animals. Cobbett $^{3}$, recovering the non-pathogenic bacilli from the tiny abscess produced by their inoculation, also tried the effect of passage through guinea-pigs-once four animals in succession-with negative result.

Returning now to the question of the significance of the diphtheria bacillus in the throats of scarlet fever patients on admission to hospital, and regarding it in the light of these investigations, we must, I think, conclude that in large centres of population, where diphtheria always exists, these organisms are to be found in a not inconsiderable proportion of school-children; that, in the absence of evidence of clinical diphtheria and of a history of exposure to that affection, the bacilli are, however, in the majority of cases of the non-virulent or saprophytic type and, therefore, of little hygienic importance; that in cases, on the other hand, where the clinical supports the bacteriological examination the bacilli are almost certainly virulent, and therefore dangerous; while in cases where the patient is known to have been

1 Path. Soc. of Lond. Trans, 1898.

2 Zeitschr. f. Hygiene, Bd. xxxv., p. 87.

3 Journal of Hygiene, r., p. 497. 
exposed to infection the chances are great that the organisms are of the pathogenic variety, and such cases should always be regarded with grave suspicion.

\section{Diphtheria Bacilli in the Nasal Cavities.}

Apart from the subject of fibrinous rhinitis, the literature in relation to the presence of diphtheria bacilli in the nose is by no means extensive.

(i) Among scarlet fever patients on admission into hospital.-From the noses of 420 patients admitted into this hospital certified scarlet fever, cultures were made with a view to ascertaining the prevalence of bacilli morphologically resembling the diphtheria bacillus. Owing to the luxuriant growth of other organisms found in the noses of scarlet fever patients, the character of the culture often did not admit of the satisfactory examination of single colonies, while the frequent presence of Hoffman's bacillus considerably increased the difficulty.

Clinically, two patients were suffering from faucial diphtheria without evidence of scarlet fever; diphtheria bacilli were found in the throats of both, but in the nose of one only.

One patient had scarlet fever complicated with laryngeal diphtheria: the Klebs-Loeffler bacillus was obtained from both throat and nose.

In two cases of scarlet fever, examination of the nasal cavities in the receiving room revealed the presence of well-defined membrane, limited to the Schneiderian lining and adherent to septum and inferior turbinated bones. Diphtheria bacilli were obtained from throat and nose.

One child came with the history that her two sisters were in the hospital suffering from diphtheria. Cultures from the throat were negative, but diphtheria bacilli were obtained from the nose, although the nasal cavities appeared quite normal. The bacilli were found to kill guinea-pigs in 48 hours in the usual dose of broth culture; when antitoxin was simultaneously injected the guinea-pig remained unaffected.

The remaining 414 cases presented on careful inspection no evidence of either faucial diphtheria or fibrinous rhinitis; nevertheless, from the nasal cavities of 33 were obtained bacilli, morphologically indistinguishable from the Klebs-Loeffler bacillus. In 10, the organisms were found in both throat and nose; bacilli from the noses of two of these, and from the throat of another, were tested and found to be non-virulent. In 23, the bacilli were present in the nose only; three of these cultures were inoculated into guinea-pigs and similarly found to be non-virulent. 
Thus, in the six cases in which the pathogenicity was examined, the bacilli, although capable of producing an acid reaction in glucose broth, were found to be non-virulent to guinea-pigs.

In 234 cases, Hoffmann's bacillus was present in the nose cultures. In about thirty of these, all very mixed cultures, there were found in the films spread a few short, straight, rather thick bacilli, with uniform diameter and rounded ends, which presented fair-sized pole granules both when treated with Loeffler's stain and that of Neisser. These rods were sparsely scattered among the Hoffmann bacilli, sometimes singly but generally in small groups. Pure subcultures could not be obtained, so that it was not determined whether they were to be regarded as a variant of Hoffmann's bacillus exhibiting pole granules, or as a short type of the diphtheria organism intimately mixed with these bacilli in the specimen. The treating of them as being of no more serious import than Hoffmann's bacillus has not been followed by ill-result, but their occasional presence adds considerably to the labour of a routine bacteriological examination of the nose.

(ii) Among the general public.-Mr Lambert Lack ${ }^{1}$ made cultures from the noses of all the children attending his ear, nose, and throat out-patient practice during the first fortnight in October, and 25 children under 12 years of age attending a medical clinique-in all, 100 patients. About 40 were cases of adenoids, four had atrophic rhinitis, many had slight running from the nose, while none were seriously ill, and in no case was there a history of exposure to diphtheritic infection. In 13 per cent. the diphtheria bacillus was found, and in 52 per cent. that of Hoffmann. Unfortunately, as Mr Lack informs me, in no case was the virulence tested.

The statistics of Gross ${ }^{2}$ at the Children's Hospital at Boston, while not to be regarded as indicating the prevalence of diphtheria bacilli in children when admitted into hospital, are interesting because they confirm the above experiences as to the greater frequency of these organisms in the nose than the throat. All the cases entering the hospital during 6 months-316 in all-had culture examinations made of throat and nose, two cultures, 24 hours apart, being taken on admission and subsequently repeated once weekly as long as the cases remained in the house. Twenty-six, at one time or another, showed Klebs-Loeffler bacilli; two only of these had clinical diphtheria. Of the 314 children without clinical diphtheria, 7 had the bacillus in the throat and 17 in the nose, during some period of their stay in hospital.

1 Med. Chir. Trans., Lxxxu.

2 University Medical Magazine, Oct., 1896. 
(iii) Among nurses in attendance on diphtheria patients.-Cultures were made from the noses of 25 nurses working in our diphtheria wards; in two were found bacilli indistinguishable from the Klebs-Loeffler bacillus. The nasal cavities in each appeared normal.

(iv) In post-scarlatinal rhinorrhoea.-Chronic rhinitis with sore nostrils, a varying amount of discharge and a tendency to the formation of pustules on parts of the body, is a fairly common sequel of scarlet fever. Todd ${ }^{1}$ in 1898 called attention to the fact that this "external rhinitis" appeared to be sometimes due to the Klebs-Loeffer bacillus. Fifty-one cases associated with this bacillus occurred among 365 children under observation during 18 months at the London Fever Hospital. The affection, which appeared to be contagious, was most common at the ages of 3 and 4 years, no case occurring after 12 ; the nostrils were excoriated and crusted; discharge was usually slight and not uncommonly absent. In only three cases were diphtheria bacilli found in the fauces of the children affected; of these two were sisters, whose mother and brother were suffering from definite diphtheria, while the third had been similarly exposed to infection. During the 18 months over which these observations extended a bacteriological examination of the fauces of every patient was made before admission to the scarlet fever wards, and during this period only one case of post-scarlatinal diphtheria occurred in the hospital. In a few instances the virulence of the bacilli was tested, and they were found to be pathogenic to guinea-pigs.

It is not made apparent in the paper that the condition of the Schneiderian membrane was examined in these patients. In similar cases at the North-Eastern Hospital it is not uncommon to find that the condition is, in reality, fibrinous rhinitis. Since the exudation does not extend into the vestibule it may be overlooked unless a mirror be used to inspect the nasal cavities, while a previous syringing is often necessary in order that the view may not be obstructed by mucus. A few details of cases in which the inoculation test has been applied may be given.

A case of secondary diphtheria with membrane on the tonsils having occurred in a convalescent ward, the throats and noses of all the patients were examined. One boy, who had very slight discharge and no redness or soreness of nostril, was found to have the left side of the septum markedly congested with thin but definite membrane extending

1 Lancet, 8th May, 1898, p. 1458. 
over part of its surface. Free bleeding occurred when a portion of the membrane was removed, and cultures showed that virulent bacilli were present. The right nasal cavity appeared normal ; the throat, also, was natural and a culture from it negative. From two other cases, occurring in different wards under somewhat similar circumstances, bacilli, pathogenic to the guinea-pig and capable of neutralization by antitoxin, were obtained; these patients had rhinorrhoea with scabbiness of the nostrils, and when the nasal cavities were examined after syringing membrane was seen adhering to the turbinated bones. In all the general health remained practically unaffected, and the patients recovered without showing signs of paralysis or any throat lesion.

Yet another outbreak may be mentioned. In a convalescent scarlet fever ward, occupied chiefly by girls of five, a child who developed rhinorrhoea was found to have membrane on the septum and the roof of the bony orifice of each nasal cavity; on the left tonsil was a small area of deposit. Cultures showed the presence of diphtheria bacilli in throat and nose. The bacilli from one nostril were found to produce a strongly acid reaction in sugar broth, and 2 c.c. of a 48-hour broth culture proved lethal to a guinea-pig at the end of the second day, this result being prevented in another guinea-pig by simultaneous injection of antitoxin. In three rounds of cultures made from the other 25 patients, who were kept in bed meanwhile, no fewer than ten were found to have acquired the diphtheria bacillus. The spread of infection appeared to have been assisted by the fact that among the toys of the ward were school-slates which were used indiscriminately by all. Well-marked fibrinous rhinitis was present in two; their throats were normal, although in one diphtheria bacilli were present there also. The other eight children had diphtheria bacilli in the throat, which presented no abnormality except in one case, where there was a thin sheet of membrane on the right tonsil. In two the organisms were present in the nasal cavities also; these clinically appeared normal. Bacilli from each of the cases of fibrinous rhinitis, and from one of the healthy throats, were found to render glucose broth acid, and 48-hour broth cultures in 2 c.c. doses proved fatal to guinea-pigs within 48 hours, while in each case this effect was prevented in control animals by antitoxin.

In dealing with cases of rhinorrhoea which are not accompanied by evidence of fibrinous rhinitis, although associated with the presence of diphtheria bacilli in the discharge, it must not be forgotten that a scarlet fever patient who is the host of non-virulent diphtheria bacilli 
may, of course, develop the ordinary post-scarlatinal rhinorrhoea. Hence, isolated cases occurring in wards may well be of this nature. This view is borne out in an examination made by Williams ${ }^{1}$ of cases of rhinorrhoea in the scarlet fever wards of the Northern Convalescent Hospital. Unfortunately, the condition of the Schneiderian membrane was not noted in the paper. In 57 out of 141 cases of rhinorrhoea were found organisms which resembled either the Hoffmann or the diphtheria bacillus; in 36 of these the bacilli were present on reception into the Convalescent Hospital. Certain of the cultures were submitted to inoculation; of the 17 strains examined, 4 were acid-producing and virulent, 8 were acid-producing but non-virulent, while 5 were Hoffmann's bacillus.

(v) Fibrinous rhinitis under other conditions.-Lambert Lack ${ }^{2}$ found among 700 new cases attending, in one year, his clinique for ear, nose, and throat affections at the Children's Hospital, Paddington Green, 16 cases of this disease, nasal obstruction being usually the symptom for which relief was sought. During the period of his investigation Mr Lack had under his care, altogether, 36 cases; of these, 33 were under 8 years of age, while half the cases occurred during August and September. Thirty-three cases were examined bacteriologically, and in each diphtheria bacilli were found; 23 cultures were tested for virulence with positive result.

Although this affection is more common in young children it is not confined to them. Recently a woman, aged 27 , suffering from scarlet fever, was admitted into this hospital with well-marked fibrinous rhinitis; diphtheria bacilli were found in cultures from the nose, and casts of the nasal fossae were subsequently syringed away.

I might add that I have, on several occasions, seen fibrinous rhinitis develop in patients convalescent from ordinary diphtheria some weeks after the throat was clear.

The futility of attempting to stamp out infection in any outbreak of diphtheria by bacteriological examination of the throat alone must, I think, be apparent from the above observations. The remarks made at the close of the last section, with regard to the significance of bacilli found in the throat, apply, however, equally to the organisms found in the nose.

l Brit. Med. Journ, 21st Dec., 1901, p. 1799.

2 loc. cit. 


\section{The Prevention of Post-scarlatinal Diphtheria.}

This subject divides itself naturally into two parts-the prevention of the introduction of virulent diphtheria bacilli into a ward, and the prevention of spread among patients.

(A.) Prevention of Introduction.-Introduction, as has been pointed out, seems to take place through patients who, whether suffering from clinical diphtheria or not, are admitted with virulent bacilli in their throats or noses; occasionally it may occur through members of the staff who have been working in wards containing cases of diphtheria.

(i) By members of the staff--It follows from the observations recorded in preceding sections that the transference of nurses from the diphtheria to the scarlet fever side should not occur more frequently than can be helped, and that those who have been working in wards containing diphtheria or post-scarlatinal diphtheria patients should not be put on duty in scarlet fever wards unless they have been proved by culturing to be free from the means of infecting their charges with diphtheria. It is obvious, as Denny ${ }^{1}$ remarks, that a person is dangerous in proportion to the number of bacilli which are given off from him. Thus, in an acute case of diphtheria when the child is coughing and gagging and the secretions are profuse, the bacilli will be disseminated more than they are in mild or convalescent cases. Still from mild cases, and equally from healthy individuals, there is abundant opportunity, as in coughing and sneezing, for the bacilli to be disseminated. A likely means in the circumstances under consideration, both of acquiring and of distributing infection, would seem to be the fondling and kissing of children; the rule, understood in every hospital, that no child should be kissed by a nurse is without doubt very frequently broken.

It is important that in the selection of fever nurses special attention should be paid to the condition of the throat, on account of the relation which appears to exist between it and the period during which infectivity, thus acquired, lasts. Of four nurses, who about the same time, without impairment of health, carried diphtheria bacilli in their throats, one had large, rugged tonsils with some remains of adenoids, while the throats of the others were normal in appearance. The latter were by antiseptic treatment freed from the organism in a few days. The one with the abnormal throat, in spite of over a month's rigorous local treatment, followed by some weeks at the seaside, showed virulent diphtheria bacilli nine weeks after the first examination although

loc. cit. 
during the whole of this period she had not been in contact with any source of infection.

(ii) By patients. - A careful inspection of the throat on admission, with bacteriological examination in cases of doubt, is, of course, customary at all fever hospitals. That a similar examination of the nasal cavities is almost of equal importance is, I think, evident from the series of cases examined at this hospital.

The important question arises as to whether a routine bacteriological examination of all patients should be made on admission. Such an examination of the throat was advocated by Garratt and Washbourn ${ }^{1}$ as a method of preventing post-scarlatinal diphtheria. They found, as already mentioned, that of the patients admitted under their care at the London Fever Hospital, from March, 1896, to December, $1898,1.2$ per cent. had in their throats bacilli resembling those of diphtheria. These cases were isolated. In 1896, of 637 admissions, three developed post-scarlatinal diphtheria; in 1897, of 431, one; in 1898, out of 325 patients, none at all ; the previous record for the hospital had been, in 1893, of 764 admissions, four; in 1894, of 294, one; and in 1895, when, it is stated, an outbreak necessitated the closing of some wards, fourteen. Nevertheless, $\operatorname{Todd}^{2}$ found that this systematic examination of the throats had not prevented the occurrence, in the same wards, of a contagious rhinitis associated with the presence of diphtheria bacilli, which in several cases were pruved to be virulent; a circumstance which would suggest the advisability of a similar routine examination of the nose on admission.

In the previous sections, however, the attempt has been made to show that, where the patient brings no history of exposure to diphtheria and presents no clinical evidence of that affection in either throat or nose, the bacilli which may be present are in most cases of a saprophytic and harmless character. It must also be remembered, to look at the question from another standpoint, that a single negative culture is no proof of the absence of diphtheria bacilli. Dr Hill, the Director of the Boston Board of Health Laboratory, states, with regard to bacteriological examination in suspected cases of diphtheria, that from 5 to 10 per cent. of the cases finally proving positive fail to yield a positive result at the first examination. In the examination of cases admitted into this hospital certified diphtheria, a similar error of about 5 per cent. has been noted in cases where the character of the throat has led to the

1 loc. cit.

2 loc. cit.

3 Boston Med. and Surg, Journ., 7th March, 1901. 
belief that the bacilli finally found have not been acquired in hospital. It appears probable from this that a routine examination by single culture would fail to detect quite an appreciable proportion of the carriers of diphtheria bacilli. Thus the adoption of this method would, on the one hand, lead to the isolation of many patients with harmless bacilli, while, on the other, it would not altogether prevent the admission of patients carrying virulent organisms. That it would lead to greater accuracy in clinical observation is probable, but whether the very considerable labour which a routine examination of the throat and nose entails, when dealing with the large numbers which these hospitals receive, would, on this score, be sufficiently repaid is, at least, open to doubt. In considering the subject it is impossible to avoid being influenced by the fact that post-scarlatinal diphtheria has in recent years ceased to have that dread influence over the course of scarlet fever which it formerly possessed, and that now the cases in which it at all assists in producing the fatal result form but an infinitesimal proportion of the patients treated in these hospitals. As already remarked, at this hospital in 1901 of 3,094 scarlet fever patients under treatment only one died who had suffered from post-scarlatinal diphtheria, and he died of lobar pneumonia a month after the commencement of his attack.

Although belonging more strictly to the next subdivision, another suggestion may here be noticed,--the injection of a prophylactic dose of antitoxin. Park states ${ }^{1}$ that at the Foundling Hospital, New York, where in the past diphtheria had so frequently followed measles, it is now the rule, instead of making cultures regularly, to inmunize every case with antitoxin so soon as the Koplik spots are noticed. Since the starting of this procedure diphtheria as a complication of measles has been completely eliminated. Professor W. R. Smith ${ }^{2}$, in the Harben Lectures for 1900, speaking of prophylactic doses of antitoxin, suggested their use "in hospitals in the case of those scarlet fever patients exposed to the risk of diphtheritic infection, and who so frequently acquire it, especially in the case of all those sent to the convalescent hospitals." In the case of scarlet fever patients, however, there are many objections to this method,-among others, the length of the period of isolation, averaging ten weeks, in relation to the often temporary character of the immunity conferred by antitoxin, and the important fact that, by preventing the development of the disease

1 Trans. of the Assoc. of Amer. Physicians, xv., 1900, p. 222.

2 Joumal of State MLedicine, viII., p. 182, March, 1900. 
it encourages a free distribution of virulent bacilli among the protected patients which on their discharge may work havoc among others.

(B.) Prevention of spread.-While bacteriological investigation may, I think, be made subsidiary to the clinical examination in attempting to prevent the introduction of infection, in the prevention of spread, on the other hand, there can be no doubt it should take a very prominent part. When the virulent bacillus has invaded a scarlet fever ward, as evidenced by the occurrence of a case of secondary diphtheria, all the patients should be kept in bed and inter-infection by their toys, handkerchiefs, etc., prevented, while the condition of their throats and nasal cavities is investigated, two rounds of cultures, at least, being made from nose and fauces. Similar cultures should also be made from the nurses. Those in whom diphtheria bacilli are found should be removed to an isolation ward and appropriately treated. When this method is adopted, a ward can usually be safely considered free from infection after only a few days' quarantine. Attention has been directed to the importance of an examination of the nurses and of a supervision over the toys (especially with regard to the slates and mouth-instruments, so frequently supplied by parents), because in several examinations in our wards, in connection with cases of post-scarlatinal diphtheria, these have appeared to be important factors in stamping out infection.

I now come to an important point in the administration of fever hospitals. It is obvious that the number of patients developing post-scarlatinal diphtheria will depend on the number brought in contact with those already infected with the bacillus. When resident medical officer some years ago at a hospital for children, I became convinced that in institutions of that character many-bedded wards were a mistake, on account of the liability to introduction of various infectious diseases. Experience of isolation hospitals had led me to the same opinion as regards fever hospitals. In the designing of institutions to be used largely or entirely for the treatment of children, there can be no question that the smaller the wards, consistent with economy in building and administration, the better.

It naturally follows that the intermingling of patients from different wards is much to be deprecated. At several isolation hospitals in this country a common recreation room is provided for the use of all the scarlet fever convalescents. It is easy to see how this favours the spread of secondary diphtheria: an infecting child is brought into most intimate contact with scores of more or less susceptible patients, 
and it is but natural that hospitals of such construction should present a high incidence of post-scarlatinal diphtheria. Again, it cannot be doubted that in hospitals where this general congregation of patients is permitted the production of bacillus-carrying, yet apparently healthy, children is not infrequent. The possible harm which may be done by the introduction of virulent diphtheria bacilli into a ward is to be gauged, not by the number of patients who develop post-scarlatinal diphtheria, but by the number infected with the bacillus. The former, which alone is recorded in a hospital's statistics, is no guide to the amount of evil which may possibly result from the discharge to their homes and schools of children who, though apparently healthy, carry with them the virulent bacillus of diphtheria. It follows that the adoption of what may be called a "barrack" system should be discountenanced in hospitals for fever convalescents, that the children from different wards should be prevented from mixing, and that a diligent search, after the manner suggested, should be made among those known to have been exposed to infection.

\section{Summary of Conclusions.}

1. The principal cause of post-scarlatinal diphtheria is the admission into the scarlet fever wards of patients who, whether suffering from clinical diphtheria or not, are carriers of virulent diphtheria bacilli.

2. In large centres of population, where diphtheria always exists, diphtheria bacilli are to be found in a not inconsiderable proportion of school-children. In the absence both of evidence of clinical diphtheria and of a history of exposure to that affection, the bacilli are, in the majority of cases, of the non-virulent or saprophytic type and of little hygienic importance; in cases, on the other hand, where the clinical supports the bacteriological examination the bacilli are almost certainly virulent, and therefore dangerous; while in cases where the patient is known to have been exposed to infection the chances are great that the organisms are of the pathogenic variety, and such cases should always be regarded with grave suspicion.

3. On account of the prevalence of the non-virulent bacillus and the fallacies of single cultures, it may be doubted whether a routine bacteriological examination of throat and nose of all patients on admission would prove of sufficient value to repay the labour involved. 
Chief reliance must be placed on a careful inspection on admission, not only of the throat, but also of the nasal cavities, bacteriological examination being resorted to in cases of doubt.

4. In eradicating infection from an invaded ward bacteriological methods are, on the other hand, of prime importance, since children with apparently healthy throats and noses are often found to have acquired the virulent bacillus. An attempt to stamp out any outbreak of diphtheria by bacteriological examination of the throat alone is, however, futile, owing to the frequent infection of the nasal cavities.

5. Fibrinous rhinitis, which appears to be a not infrequent, though often unrecognised, affection of children is a common form of postscarlatinal diphtheria.

6. Although the treatment of diphtheria in the same hospital has no appreciable influence on the incidence of this complication of scarlet fever, it is advisable that precautions should be taken lest nurses harbouring diphtheria bacilli carry infection from the diphtheria to the scarlet fever wards.

7. To limit the risk of exposure to infection many-bedded wards should be avoided, and the mixing of patients from different wards prevented. 